\title{
Entomofauna Associada ao Cultivo do Cubiu (Solanum sessiliflorum Dunal) no Município de Benjamin Constant, Amazonas, Brasil
}

\author{
Agno Nonato Serrão Acioli ${ }^{\bowtie}$, Grace Kelly Guimarães da Costa, Thaysa Nogueira de Moura, \\ Marcelo de Almeida Guimarães, Ronaldo de Almeida \& José Furtado de Miranda
}

Universidade Federal do Amazonas/Instituto Natureza e Cultura - UFAM/INC, e-mail: acioli@ufam.edu.br (Autor para correspondência ${ }^{\bowtie}$ ), graceguimaraes13@hotmail.com, thaysamoura@gmail.com, mguimara@ufc.br, ronaldoufam@gmail.com, mirandaufam@hotmail.com.

\section{EntomoBrasilis 7 (2): 99-105 (2014)}

Resumo. O cubiu (Solanum sessiliflorum Dunal) (Solanaceae) é originário da região Amazônica. Suas propriedades alimentares, medicinais e agronômicas têm despertado o interesse de pesquisadores desde 1970. No entanto, estudos sobre insetos em plantas tanto de ocorrência natural como de cultivos são escassos. O objetivo deste trabalho foi realizar um levantamento da ocorrência de insetos e caracterizar os danos aparentes aos cultivos de cubiu localizados em Benjamin Constant, no Alto Solimões, Amazonas, Brasil. Foram encontradas 51 espécies de insetos adultos distribuídas em 22 famílias e sete ordens. Hymenoptera, Hemiptera e Coleoptera congregam juntas 82,35\% das espécies encontradas neste estudo. Hemiptera e Coleoptera são as ordens com maior número de famílias, oito e cinco, respectivamente; enquanto a família Formicidae (Hymenoptera) tem o maior número de espécies (11). Foram registradas 18 espécies de insetos fitófagos, 10 pertencentes à ordem Coleoptera e oito à Hemiptera. O maior número de famílias foi registrado para a ordem Hemiptera com seis, para Coleoptera identificou-se apenas três. Os Coleoptera da espécie Epicauta pestifera Werner (Meloidae) constituem a principal praga do cultivo de cubiu em Benjamin Constant, pois seu ataque em reboleira é capaz de destruir várias plantas em um único dia e exterminar plantios em poucas semanas. Outros fitófagos dos gêneros Colaspis e Cerotoma, ambos Chrysomelidae, ao consumirem o tecido foliar expõem as plantas ao ataque de microrganismos e reduzem a área fotossintética. Enquanto que Edessa rufomarginata De Geer (Pentatomidae, Hemiptera) causa danos diretos e indiretos e merece atenção durante todo o ciclo de produção.

Palavras-chave: Alto solimões, injúrias; insetos-praga, morfoespécies.

\section{Entomofauna Associated to Cultivation of Cubiu (Solanum sessiliflorum Dunal) in Benjamin Constant City, Amazonas, Brazil}

Abstract. Cubiu (Solanum sessiliflorum Dunal, Solanaceae) is originally from Amazon region and its dietary, medicinal and agronomic properties sparked the interest of researchers since 1970. The studies about insects on cubiu plants both of natural occurrence as well as in cultivations, is scarce. This study aims to relate the visitor insect of cubiu plants, with emphasis to the phytophagous species. We found 51 species of the mature insect, distributed among 22 families and seven orders. Hymenoptera, Hemiptera and Coleoptera represent together 82.35\% of the species found in this study. Coleoptera and Hemiptera are the orders with larger number of families, eight and five, respectively, while Formicidae (Hymenoptera) is the family with the largest number of species (11). We recorded 18 species of phytophagous insects, ten belong to the order Coleoptera and eight to the order Hemiptera. However, the largest number of families was recorded to the order Hemiptera with six, while Coleoptera has only three. The species of beetles Epicauta pestifera Werner (Meloidae) constitute the main pest of the cultivation of cubiu in Benjamin Constant, because the attack in agglomerates may destroy many plants in just one day and exterminate the plantation within weeks. Other genus of phytophagous such as Colaspis and Cerotoma, both Chrysomelidae, consumes foliar tissues, exposing the plants to the attack of microorganisms, which may compromise the photosynthetic capacity of the plant. Edessa rufomarginata De Geer (Pentatomidae, Hemiptera) causes direct and indirect damages and deserves attention throughout the production cycle.

Keywords: High Solimões, injuries; Insect pests; morphospecies.

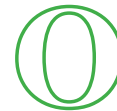
cubiu (Solanum sessiliflorum Dunal), pertencente à família Solanaceae, é uma hortaliça-fruto bastante difundida entre os povos indígenas e tradicionais da região Amazônica. É originário da região do alto Orinoco da bacia Amazônica, podendo ser encontrado em toda a Amazônia brasileira, peruana, equatoriana, colombiana e venezuelana (Silva-Filho 1998), tanto em condições subespontâneas como em forma cultivada (SıLva-Filho et al. 1997). Na alimentação o fruto pode ser consumido in natura ou processado na forma de sucos, doces, geleias, compotas e, na culinária, é utilizado em pratos à base de peixe, carne, frango e misturado com saladas (PAHLEN 1977). É uma importante fonte de fibra alimentar, macro e micro nutrientes, pectina e vitamina C (Yuyama et al. 2002, 2007; Pires et al. 2006). Segundo Silva-Filho (1998), o cubiu é considerado um importante recurso genético da Amazônia, com potencial para uso na agroindústria e em programas de segurança alimentar para populações da região Amazônica.
O cultivo do cubiu na região do Alto Solimões, tríplice fronteira Brasil-Peru-Colômbia, é realizado de forma intensa e tradicional, sem aplicação de defensivos agrícolas. No entanto, a presença constante e crescente de insetos nesses cultivos tem preocupado pequenos agricultores que utilizam esta olerícola, em seus policultivos, com a finalidade de incrementar sua renda. Durante as observações de campo, verificou-se que tanto as populações de cubiu que ocorrem naturalmente como as cultivadas intencionalmente são atacadas por várias espécies de insetos fitófagos.

Para a Amazônia brasileira, em estudos realizados na região de Manaus, sobre a ocorrência de insetos no cultivo do cubiu, Couturier (1988) cita a ocorrência de 14 espécies nocivas ou

Agência(s) de Financiamento: CNPq (projeto MCT/CNPq/CTAmazônia No 55/2008) 
potencialmente nocivas e destaca a necessidade de prestar mais atenção para Corythaica cyathicollis (Costa) (Tingidae, Hemiptera), Planococcus pacificus Cox (Pseudococcidae, Hemiptera), Manduca sexta (Linnaeus) (Sphingidae, Lepidoptera), Phyrdenus muriceus Champion (Curculionidae, Coleoptera) e uma espécie não identificada de Cryptorhynchinae (Curculionidae, Coleoptera). Mais recentemente, Delgado et al. (2011), em estudos realizados na Amazônia peruana, acrescentam a essa lista mais três espécies de insetos: Neoleucinodes elegantalis (Guenée) (Piralidae, Lepidoptera), Epicauta pestifera Werner (Meloidae, Coleoptera) e Chromacris peruviana (Pictet \& Saussure) (Acriididae, Orthoptera) e uma espécie de nematódeo do gênero Melodogyne. No geral, estes insetos causam injúrias, principalmente nas folhas e caules, podendo ocasionar elevado prejuízo econômico ao agricultor.

Baseado no exposto, este estudo tem como objetivos principais realizar um levantamento da ocorrência de insetos, bem como caracterizar os danos aparentes provocados por insetos fitófagos aos cultivos de cubiu localizados em região de terra firme do município de Benjamin Constant, região do Alto Solimões, Amazonas, Brasil.

\section{MATERIAL E MÉTODOS}

Local de Estudo. O estudo foi desenvolvido em região de terra firme no Município de Benjamin Constant (04 ${ }^{\circ} 22$ '59"S/ $70^{\circ} 01$ '52"W), com altitude média de $70 \mathrm{~m}$ acima do nível do mar e localizada na mesorregião do Alto Solimões, sudoeste do Estado do Amazonas.

O clima predominante na região Amazônica é do tipo equatorial (superúmido, Af segundo a classificação de Köppen), sem estação seca/semisseca, caracterizado por elevado índice pluviométrico, podendo variar na mesorregião do Alto Solimões de $2.500 \mathrm{~mm}$ até $3.600 \mathrm{~mm}$, sendo a média anual de $2.562 \mathrm{~mm}$. A temperatura média anual é de $25,7^{\circ} \mathrm{C}$ (Coelho et al. 2005).

Estudos de solos realizados em uma área de $9.000 \mathrm{~m}^{2}$ realizado próximos a cidade de Benjamin Constant demonstraram que as classes de solos predominantes são os Cambissolos (90\%) e os Gleissolos (10\%) de idades relativas bem mais jovens em relação àqueles profundos e bem drenados de grande parte das terras firmes da região Amazônica (CoElho et al. 2005).

Material e Procedimentos. As sementes de cubiu foram adquiridas de frutos maduros comprados diretamente dos agricultores que comercializam sua produção na feira municipal de Benjamin Constant, AM. As sementes foram retiradas dos frutos, lavadas em água corrente e secas ao ar livre.

Os sacos para semeadura, com capacidade para $1 / 2 \mathrm{~kg}$, foram preenchidos com solo peneirado e cama de frango na proporção 1:1, posteriormente esse substrato foi umedecido e foram feitas pequenas covas com profundidade de aproximadamente 0,02 $\mathrm{m}$, na qual as sementes foram semeadas e cobertas por uma fina camada desse substrato. O transplantio das mudas para o local definitivo foi realizado quando as plantas apresentaram três a quatro folhas definitivas.

$\mathrm{Na}$ área de cultivo, o solo foi caracterizado como do tipo argiloso, em declive, sombreado e coberto predominantemente por gramíneas. Foram abertas 28 covas, cada uma media aproximadamente $0,20 \times 0,20 \times 0,20 \mathrm{~m}$ e distantes entre si $1 \mathrm{~m}$. Em cada cova o solo superficial foi separado, com a finalidade de ser misturado a um composto de cama de frango e areia na proporção 1:1. Para cada cova adicionou-se ao solo superficial uma quantidade de composto suficiente para seu preenchimento. Foi plantada uma planta/cova e para manter a área limpa, periodicamente foram feitas capinas manuais. Nenhum tipo de defensivo agrícola foi usado durante o período deste estudo.

Além das coletas na área de cultivo experimental citada acima, também foram feitas observações de plantios de cubiu em pequenos sítios localizados nos quilômetros 7, 15 e 17 da BR-307, rodovia que liga os municípios de Benjamin Constant a Atalaia do Norte, ambos no Amazonas.

Captura e Identificação dos Insetos. As observações e coletas foram realizadas diariamente em dois horários, das 08:00 - 09:00 e das 17:00 - 18:00 h, durante cinco meses (novembro/2011 a março/2012), período considerado chuvoso na mesorregião do Alto Solimões.

Durante as coletas as plantas foram cuidadosamente vistoriadas e os insetos foram capturados com o auxílio de uma pinça entomológica e/ou frascos de boca larga. Os insetos coletados foram conservados em recipientes, devidamente etiquetados e preservados em álcool 70\%. Além da coleta, foram feitas observações de campo referente à parte da planta em que o inseto foi coletado, qual e como era o tipo de dano causado à planta no momento da coleta.

Os insetos foram examinados sob estereoscópio com capacidade de aumento de até 45x. A identificação taxonômica foi realizada com base em chaves entomológicas disponíveis em GAllo et al. (2002), Triplehorn \& Jonnson (2011), Rafael et al. (2012), bem como em chaves e descrições mais específicas para gêneros e espécies. Todo o material entomológico coletado foi depositado na Coleção de Insetos de Ciências Agrárias - CICA - do Instituto Natureza e Cultura/Universidade Federal do Amazonas - INC/ UFAM, em Benjamin Constant - Amazonas.

\section{RESULTADOS E DISCUSSÃO}

Diversidade de insetos. Foram encontradas 51 espécies/ morfoespécies de insetos adultos sobre os cultivos de cubiu, distribuídas em 22 famílias e sete ordens (Figura 1). As ordens Hymenoptera, Hemiptera e Coleoptera representaram 82,35\% das espécies encontradas neste estudo. Estas três ordens são reconhecidamente classificadas como megaordens, devido ao elevado número de espécies, que de forma geral ocupam vários ambientes, que podem ser naturais ou antrópicos (GALLO et al. 2002; RAFAEL et al. 2012). Neste estudo foi registrado que Hemiptera e Coleoptera são as ordens com maior número de famílias, oito e cinco, respectivamente, enquanto Formicidae (Hymenoptera) é a família que apresentou o maior número de morfoespécies (11). Por outro lado, as ordens Diptera, Lepidoptera, Blattaria e Neuroptera constituem apenas 17,65\% das espécies. Para a ordem Diptera são registradas três famílias, enquanto que para as demais ordens apenas uma família.

A ordem Hymenoptera foi a que apresentou maior diversidade de espécies/morfoespécies (17), o que corresponde a 33,33\% do total, sendo estas distribuídas em apenas três famílias: Formicidae, o grupo mais abundante com $64,71 \%$ das espécies, seguido por Vespidae com $23,52 \%$ e Chalcididae com $11,77 \%$. Em estudos sobre a fauna de Hymenoptera associada ao cultivo do jiló (Solanum gilo Raddi) PICANÇO et al. (1997) identificaram oito famílias, sendo que as mais abundantes foram Formicidae e Vespidae com nove e seis espécies, respectivamente. Todos os Hymenoptera registrados neste estudo formam um importante grupo de insetos que podem ser utilizados em programas de manejo ecológico ou manejo integrado de pragas (GALLo et al. 2002; TAVARES \& ARAUJo 2007), pois Formicidae e Vespidae são predominantemente espécies predadores enquanto Chalcididae são parasitóides.

Hemiptera foi o segundo grupo com maior diversidade de insetos encontrados no estudo com 13 (25,49\%) espécies, sendo composto predominantemente por espécies fitófagas. Das oito famílias registradas, para esta ordem, somente Reduviidae é considerada predadora. BAsso et al. (1974), na região Sul do Brasil, relacionaram várias espécies fitófagas da ordem Hemiptera atacando diversas espécies botânicas cultivadas ou não do gênero Solanum. Os hemípteros fitófagos encontrados neste trabalho 
são pragas potenciais, que dependem de diversos fatores para alcançarem tal status, o que torna importante o seu registro nos cultivos observados.

Para a ordem Coleoptera, cinco famílias foram encontradas neste estudo, sendo todas elas consideradas fitófagas. Chrysomelidae foi a que apresentou o maior número de representantes, com $50 \%$ das espécies encontradas, sendo a outra metade distribuída de forma igualitária (12,50\%) entre as famílias. Para o gênero Solanum, BAsso et al. (1974) já havia registrado as famílias Chrysomelidae, Curculionidae e Meloidae, enquanto Couturier (1988) registrou para o cubiu espécies das famílias Chrysomelidae e Curculionidae. No entanto, até o presente momento não havia registros da presença de espécies da família Nitidulidae, o que foi observado neste estudo.

Das famílias de Diptera encontradas, destaque pode ser feito para as espécies pertencentes à Ropalomeridae. Pois, conforme MARQues et al. (2004), as informações sobre biologia, ecologia e hábitos da espécies são escassos. Este é o primeiro registro de espécies dessa família sobre o cultivo de cubiu, até então as espécies de Ropalomeridae registrados na Amazônia, foram em pomares de espécies frutíferas próximo à região de Manaus (MArques et al. (2004). Para a ordem Neuroptera foi registrada apenas uma morfoespécie pertencente à família Chrysopidae, considerada muito comum na região, sendo pertencente a um importante grupo de insetos predadores que podem ser utilizados em manejo de pragas.

Insetos fitófagos em cultivos de cubiu no município de Benjamin Constant. Os insetos fitófagos registrados neste trabalho perfazem um total de 18 espécies pertencentes a nove famílias, sendo três da ordem Coleoptera e seis da ordem Hemiptera (Tabela 1). Essas duas ordens contêm as principais espécies de insetos que causam injúrias às plantas de cubiu, resultados esses que corroboram com os estudos de Couturier (1988) e Delgado et al. (2011). Vale ressaltar que 16 espécies são novos registros para o cultivo de cubiu, elevando dessa forma

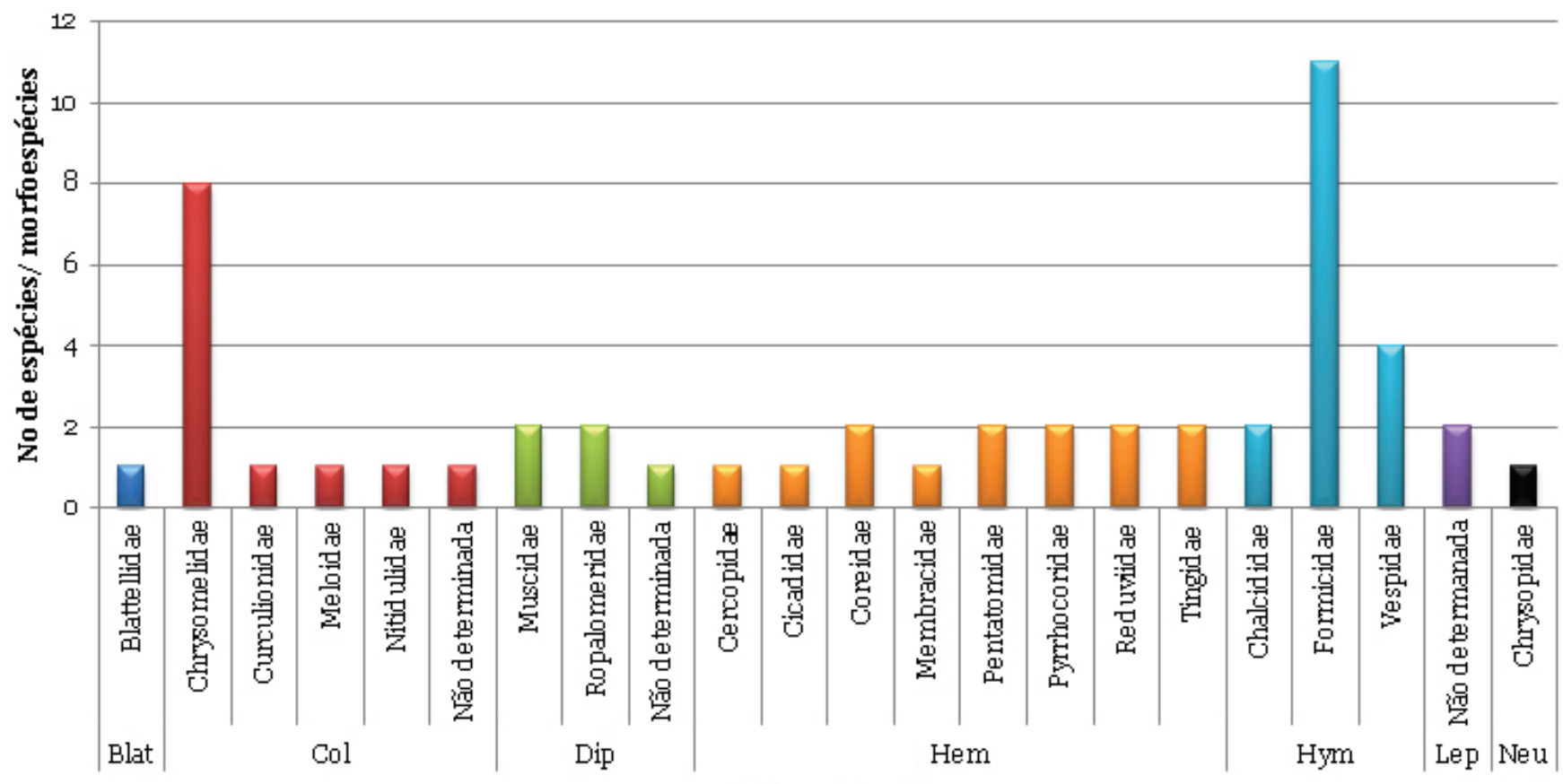

Ordens/Famílias

Figura 1. Diversidade de insetos coletados sobre o cultivo do Cubiu (S. sessiliflorum, Solanaceae) no município de Benjamin Constant, na região do Alto Solimões, Amazonas, Brasil. Blat = Blattaria, $\mathrm{Col}=$ Coleoptera, Dip = Diptera, Hem = Hemiptera, Hym = Hymenoptera, Lep = Lepidoptera, Neu $=$ Neuroptera.

para 33 o número de espécies de insetos fitófagos do cubiu.

Família Chrysomelidae. Foram coletados os adultos de oito espécies da família Chrysomelidae (Tabela 1) se alimentando sobre as folhas das plantas do cubiu. Ao se alimentarem, estes crisomelídeos podem causar danos superficiais no tecido foliar ou mesmo perfurar as folhas fazendo orifícios de formato mais ou menos regulares e circulares (Figura 2). Seu ataque é intenso, a folha fica completamente perfurada, o que reduz sua capacidade fotossintética e, consequentemente sua produção de frutos. $\mathrm{O}$ ataque pode ocorrer em qualquer fase ou período de desenvolvimento das plantas. No entanto, acontece preferencialmente em plantas e folhas mais jovens, sendo necessário maior cuidado contra o ataque dos crisomelídeos nas primeiras semanas de vida das plantas. Parte dos Chrysomelidae constituem um importante grupo de insetos fitófagos que se alimentam de uma grande variedade de plantas, incluindo as da família Solanaceae (GALlo et al. 2002), família do Cubiu. Além de folhas, os crisomelídeos adultos também consomem raiz, caule, flores, pólen e anteras, causando danos diretos, ou podendo atuar como transmissores de vírus para as plantas, causando danos indiretos (Audino et al. 2007; Delgado et al. 2011).
Foi observado que os adultos de Cerotoma tingomarianus Bechyné se alimentam das folhas das plantas do cubiu, causando injúrias que podem comprometer o desenvolvimento da planta. $\mathrm{Na}$ região Norte do Brasil, essa mesma espécie é uma das principais pragas desfolhadoras do cultivo do feijão e os estudos indicam que os danos ocorrem em todos os estágios de desenvolvimento da planta, e o ataque severo reduz consideravelmente a produtividade (FAzOLIN et al. 2001; FAzOLIN \& EsTrela 2004). Deve-se salientar que este é o primeiro registro de ataque desta espécie em plantas de cubiu, no entanto, sua baixa incidência populacional nas plantas a torna uma praga de pouca importância para o cultivo.

Foram encontradas três espécies do gênero Colaspis se alimentando de tecidos foliares (Figura 2A). As espécies do gênero Colaspis são reconhecidamente pragas de várias famílias botânicas de interesse agrícola como Myrtaceae (eucaliptos, jaboticabeiras, goiabeiras), Malvaceae (cacaueiros), Palmae (coqueiros) (Costa-Lima 1955), Leguminosae (soja) e Solanaceae (Basso et at. 1974; Rossetto \& NAgai 1980; Sosa-Gómez et al. 2010). As espécies do gênero Colaspis também ocorrem em plantas nativas sem interesse econômico, como observado por 
Tabela 1 - Relação dos insetos fitófagos, estágio do desenvolvimento e local do dano causado em plantas de cubiu no município de Benjamin Constant, na região do Alto Solimões, Amazonas, Brasil.

\begin{tabular}{|c|c|c|c|c|}
\hline Ordem & Família & Espécie / morfoespécie & Estágio do inseto & Local do dano \\
\hline \multirow{10}{*}{ Coleoptera } & \multirow{8}{*}{ Chrysomelidae } & Cerotoma tingomarianus Bechyné* & \multirow{10}{*}{ Adulto } & \multirow{9}{*}{ Folhas } \\
\hline & & Colaspis cf. aerea Lefevre & & \\
\hline & & Colaspis sp. $\mathrm{A}^{*}$ & & \\
\hline & & Colaspis sp. $\mathrm{B}^{*}$ & & \\
\hline & & Disonycha sp. A* & & \\
\hline & & Chrysomelinae sp. $\mathrm{A}^{*}$ & & \\
\hline & & Galerucinae sp. $A^{*}$ & & \\
\hline & & Galerucinae sp. B* & & \\
\hline & Curculionidae & Curculionidae sp. $A^{*}$ & & \\
\hline & Meloidae & Epicauta pestifera Werner & & Folhas, ramos tenros e brotos \\
\hline \multirow{8}{*}{ Hemiptera } & Cercopidae & Cercopidae sp. $A^{*}$ & \multirow{8}{*}{ Ninfa e adulto } & \multirow{3}{*}{ Folhas } \\
\hline & Cicadellidae & Typhlocybinae sp. A* & & \\
\hline & Membracidae & Cyphonia sp. A* & & \\
\hline & \multirow{2}{*}{ Pentatomidae } & Pentatomini sp. $A^{*}$ & & \multirow{2}{*}{ Folhas, pecíolo de folhas e caules } \\
\hline & & Edessa rufomarginata De Geer. & & \\
\hline & Pyrrhocoridae & Dysdercus sp. A* & & \multirow{3}{*}{ Folhas e pecíolo de folhas } \\
\hline & \multirow{2}{*}{ Tingidae } & Corythucha sp. A* & & \\
\hline & & Teleonemia sp. $\mathrm{A}^{*}$ & & \\
\hline
\end{tabular}

\footnotetext{
* $=$ Novo registro.
}

REIs et al. (2001), em Viçosa - MG, em plantas produtoras de látex da espécie Oxypetalum mexiae Malme (Asclepiadaecea). No entanto, no caso específico do cubiu, Couturier (1998) destaca que a herbivoria da espécie Colaspis aff. aerea Lefévre não causa grandes danos, devido à ocorrência de ataques isolados. Entretanto, vale ressaltar que neste estudo foi registrado aumento do número de espécies e de indivíduos, merecendo este gênero maior atenção.

A espécie Disonycha sp. A, outro inseto desfolhador, também tem preferências por plantas e folhas mais jovens de cubiu. O gênero Disonycha é amplamente distribuído nas América do Norte e do Sul (Medvedev 2004). A espécie de maior interesse como praga é Disonycha glabrata (Fabricius), que já foi reconhecida como praga do cultivo do maracujá na Venezuela (HAdDa et al. 1970) e de Amarantus retroflexus Linnaeus nos Estados Unidos (Tisler 1990).

Família Meloidae. Foi observado na BR-307, nos quilômetros 14 e 15, o ataque de E. pestifera em cultivos de duas solanáceas (cubiu e pimentão). O ataque destes besouros costuma ocorrer nas primeiras e últimas horas do dia, quando a incidência solar diminui. Bandos contendo centenas de indivíduos pousam sobre as plantas alvos e começam a se alimentar vorazmente das folhas, ramos tenros e brotações, deixando somente as hastes e os frutos das plantas (Figura 2B). Constatou-se que este tipo de ataque, em reboleiras, em um único dia foi capaz de destruir uma grande quantidade de plantas. O gênero Epicauta é conhecido no Brasil como "vaquinha" das solanáceas, justamente devido a preferência alimentar por esta família botânica (CostA-LimA 1955). Delgado et al. (2011) registram o ataque dessa espécie em cultivos de cubiu na Amazônia peruana, mas para o Brasil este é o primeiro registro.

Família Pentatomidae. A espécie Edessa rufomarginata De Geer é muito comum nos plantios regionais de cubiu, sendo normal a ocorrência de vários indivíduos em uma única planta (Figura 3). Se alimenta da seiva, sugada do pecíolo das folhas e de regiões apicais do caule. Essa espécie é frequente em plantas de cubiu na região Amazônica e não causa danos
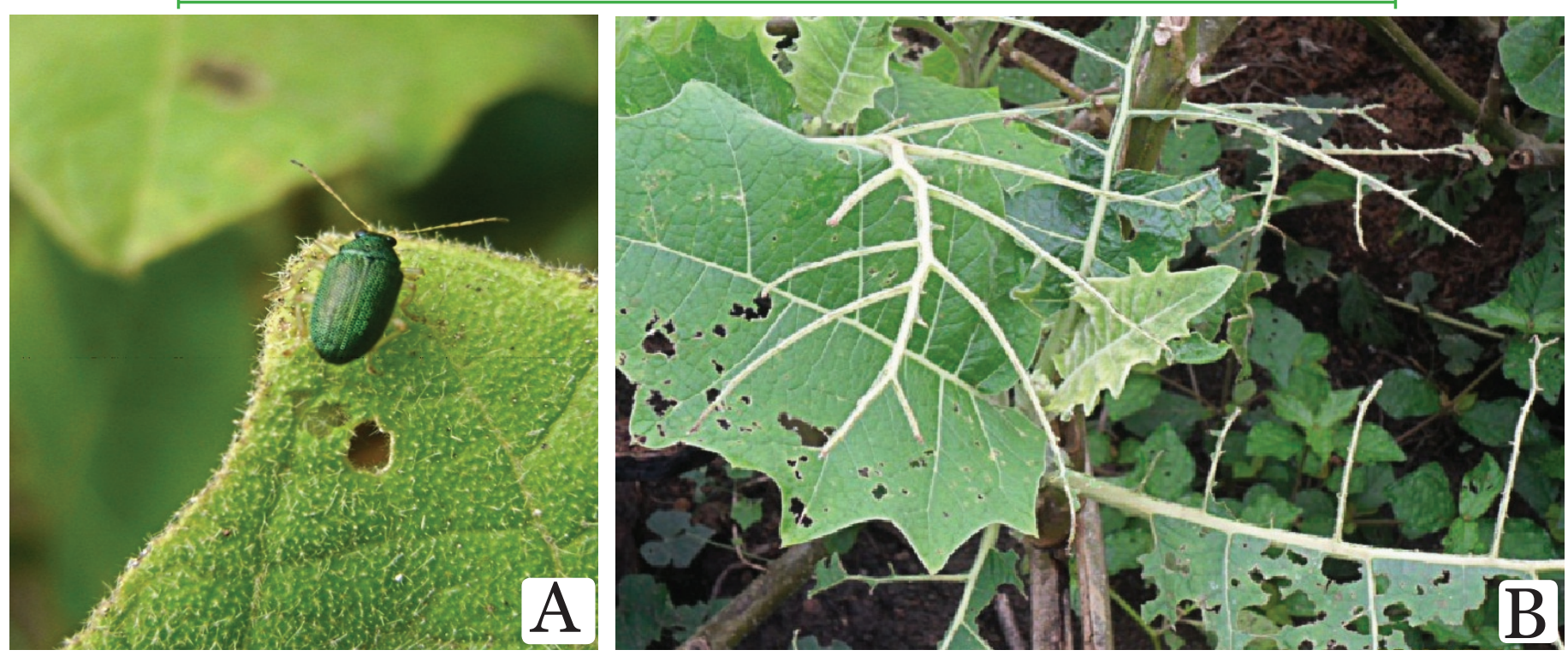

Figura 2. Injúrias causadas por Colaspis cf. aerea Lefevre (A) e Epicauta pestifera (B) em plantas de cubiu no município de Benjamin Constant, na região do Alto Solimões, Amazonas, Brasil. Fonte: A. Acioli. 
diretos de importância econômica, pois os frutos não são atacados (Couturer 1998; Delgadoet al. 2011). Outra espécie, morfotipada como Pentatomini sp. A, foi encontrada em baixa frequência na face superior das folhas e no pecíolo das plantas de cubiu. Assim como no caso da espécie E. rufomarginataDe Geer. . A, não foram observados danos que comprometessem o cultivo. Entretanto, pentatomídeos são insetos que podem atuar como vetores de doenças viróticas entre seus hospedeiros (GALLO et al. 2002).

Família Tingidae. Foram encontradas duas espécies para a família Tingidae, uma pertencente ao gênero Corythucha (Corythucha sp. A) e outra ao gênero Teleonemia (Teleonemia sp. A). A espécie Corythaica cyathicollis Costa parece ser frequente em plantas de cubiu, pois foi registrada tanto por Couturier (1998), na região de Manaus, como também por Delgado et al. (2011), na Amazônia peruana. Espécies pertencentes aos dois gêneros encontrados neste trabalho são pragas de plantas ornamentais, silvestres e agrícolas na Europa, Ásia e América do Norte (Dioli et al. 2007; Ju et al. 2011; TATU \& TAUSAn 2011; Triplehorn \& Jonnson 2011); na América do Sul poucos são os trabalhos abordando a importância desses gêneros como pragas (Neciosup \& OJeda 1973; Montemayor 2009; Varón et al. 2010).

No Brasil, o gênero Corythucha ainda não foi registrado em cultivos agrícolas de importância econômica e os principais livros de entomologia não o catalogam como praga (Costa-Lima 1955; GALLO et al. 2002). No entanto, em países fronteiriços na região Norte com o Brasil, como Colômbia e Peru, já existem registros de espécies de Corythucha causando danos econômicos a cultivos de interesse ornamental, silvestre e agrícola (Neciosup \& OJEDA 1973; VARÓn et al. 2010). Os trabalhos com o gênero Teleonemia, também são restritos, mas Broglio et al. (2012) registraram ataque severo de Teleonemia morio (Stål), em árvores de Annona squamosa Linnaeus (ata, pinheira ou fruta do conde) na região Nordeste do Brasil. Portanto, esta é a primeira vez em que espécies dos gêneros Corythucha e Teleonemia são registradas hospedados plantas de cubiu.

Família Pyrrhocoridae. Foi encontrada uma espécie de Pyrrhocoridae, Dysdercus sp. A, sugando a seiva das folhas das plantas de cubiu. O gênero Dysdercus é praga principalmente das espécies botânicas da família Malvaceae (CostA-Lima 1940; Costa-Lima et al. 1962; Schaefer 1998), principalmente do algodoeiro no qual pode causar danos diretos e indiretos (GALLO et al. 2002). Ocorre em praticamente toda a América do Sul e seu registro é assinalado para o Estado do Amazonas (Costa-Lima et al. 1962). Este é o primeiro registro de Dysdercus hospedados e se alimentando de plantas de cubiu (Solanaceae).

Estudos sobre insetos associados ao cultivo do cubiu na região Amazônica limitam-se à região de Manaus, localizada a leste do Estado do Amazonas, e a Amazônia peruana. No presente estudo, realizado em Benjamin Constant, registrou-se, pela primeira vez, exemplares das espécies pertencentes às famílias Blattellidae (Blattaria), Nitidulidae (Coleoptera), Muscidae e Ropalomeridae (Diptera), Cercopidae, Cicadelidae, Membracidae, Pyrrhocoridae e Reduviidae (Hemiptera), Chalcididae, Vespidae e Formicidae (Hymenoptera) e Chrysopidae (Neuroptera). Entre os insetos

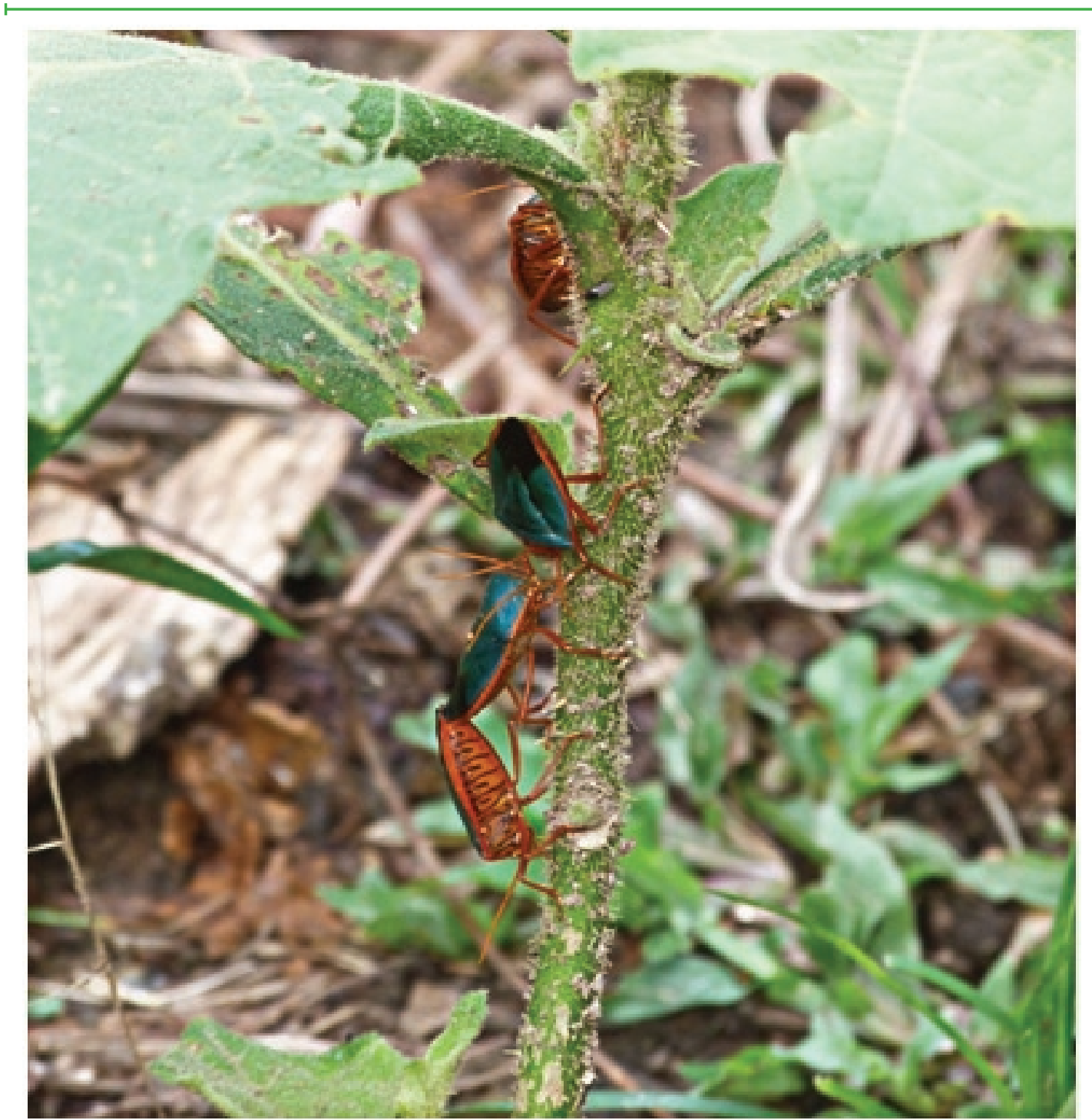

Figura 3. Edessa rufomarginata De Geer se alimentando no caule de cubiu, município de Benjamin Constant, na região do Alto Solimões, Amazonas, Brasil. Fonte: A. Acioli 
fitófagos este é o primeiro registro para os gêneros Corythucha e Teleonemia (Hemiptera: Tingidae), Disdercus (Hemiptera: Pyrrhocoridae) e a espécie $C$. tingomarianus (Coleoptera: Chrysomelidae) causando algum tipo de injúrias em plantas de cubiu. Também constatou-se a presença dos gêneros Colaspis (Coleoptera:Chrysomelidae)eEdessa(Hemiptera:Pentatomidae), ambos já registrados na região de Manaus. Mas, em Benjamin Constant, E. pestifera (Coleoptera: Meloidae) é a principal praga do cultivo de cubiu, pois o ataque em reboleira é capaz de destruir várias plantas em um único dia e exterminar todo o plantio em semanas. Outros fitófagos que merecem atenção são as espécies dos gêneros Colaspis e Cerotoma, que ao consumirem o tecido foliar expõe as plantas ao ataque de microrganismos e podem comprometer a capacidade fotossintética da planta. Já a espécie E. rufomarginata A (Pentatomidae, Hemiptera) causa danos diretos e indiretos (transmissores de doenças, principalmente vírus) e merece atenção durante todo o ciclo de produção.

\section{AGRADECIMENTOS}

O primeiro autor agradece ao Conselho Nacional de Desenvolvimento Científico e Tecnológico (CNPq) pelo apoio financeiro através do projeto de pesquisa $\mathrm{MCT} / \mathrm{CNPq} / \mathrm{CT}-$ Amazônia $\mathrm{N}^{\circ}$ 55/2008.

\section{REFÊRENCIAS}

Audino, L.D., J.M. Nogueira, P.G. Silva, M.Z. Neske, A.H.B. Ramos, L.P. Moraes \& M.F.S. Borba, 2007. Identificação dos coleópteros (Insecta: Coleoptera) das regiões de Palmas (município de Bagé) e Santa Barbinha (município de Caçapava do Sul), RS. Bagé: Embrapa (Documentos, 70), 92p.

Basso, I.V., D. Link \& O.J. Lopes, 1974. Entomofauna de algumas solanáceas em Santa Maria, RS. Revista do Centro de Ciências Rurais, 4: 263-270.

Broglio, S.M.F., M.S. Dias-Pini, L.A.A. Costa \& E.E.P. Lemos, 2012. First report and morphological redescription of Teleonemia morio (Stål) (Hemiptera, Tingidae) in Annona squamosa L. (Annonaceae) in Brazil. Revista Brasileira de Entomologia, 56: 122-124.

Coelho, M.R., L.C.C. Fidalgo, F.O. Araújo, H.G. Santos, M.L.M. Santos, D.V. Pérez \& F.M.S. Moreira, 2005. Levantamento pedológico de uma área-piloto relacionada ao projeto BiosBrasil (Conservation and sustainable management of below-ground biodiversity: Phase I), município de Benjamin Constant (AM): Janela 6. Rio de Janeiro: Embrapa (Boletim de Pesquisa e Desenvolvimento, 68), 95p.

Costa-Lima, A.M., 1955. Insetos do Brasil: $9^{\circ}$ Tomo, Capítulo XXIX: Coleópteros, $3^{\circ}$ parte. Rio de Janeiro, Escola Nacional de Agronomia, 289p.

Costa-Lima, A.M., 1940. Insetos do Brasil. $2^{\circ}$ Tomo. Capitulo XXII. Hemípteros. Escola Nacional de Agronomia, 352p.

Costa-Lima, A.M., N. Guitton, O.V.Ferreira, 1962. Sobre as espécies americanas no gênero Dysdercus Boisduval (Hemiptera, Pyrrhocoridae, Pyrrhocorinae). Memórias do Instituto Oswaldo Cruz, 60: 21-58.

Couturier, G., 1988. Alguns insetos do cubiu (Solanum sessiliflorum Dunal VAR. sessiliflorum Dunal, Solanaceae) na região de Manaus-AM. Acta Amazonica, 18: 93-103.

Delgado, C., Couturrier, G., Anteparra, M, 2011. Principales fitófagos de la cocona Solanum sessiliforum Dunal (Solanaceae) en la Amazonía Peruana. Folia Amazónica, 20: 45-51.

Dioli, P., I.G. Forini, M. Moretti \& M. Salvetti, 2007. Note sulla distribuzione di Corythucha arcuata (Insecta, Heteroptera, Tingidae) in Cantone Ticino (Svizzera), Valtellina e alto Lario (Lombardia, Italia). Il Naturalista Valtellin, 18: 59-68.

Fazolin, M., J.S. Pessoa, D.L. Amaral-Júnior, W.S.A. Oliveira \& C.R. Costa, 2001. Determinação do nível de ação para controle da vaquinha-do-feijoeiro no Acre. Rio Branco: Embrapa (Comunicado Técnico, 134), 4p.

Fazolin, M. \& J.L. Estrela, 2004. Determinação do Nível de
Dano Econômico de Cerotoma tingomarianus Bechyné (Coleoptera: Chrysomelidae) em Phaseolus vulgaris L. cv. Pérola. Neotropical Entomology, 33: 631-637.

Hadda, O.G., A. Ordosgoitty \& J. Bechyné, 1970. Daños causados por Disonycha glabrata (Fabricius) en Passiflora quadrangularis L. Agronomía Tropical, 20: 331-334.

Gallo D., O. Nakano, S. Silveira-Neto, R.P.L. Carvalho, G.C. Baptista, E. Berti-Filho, J.R.P. Parra, R.A. Zucchi, S.B. Alves, J.D. Vendramim, L.C. Marchini, J.R.S. Lopes \& C. Omotto. 2002. Entomologia Agrícola. Piracicaba, FEALQ, 920p.

Ju, R.T., F. Wang \& B. Li, 2011. Effects of temperature on the development and population growth of the sycamore lace bug, Corythucha ciliata. Journal of Insect Science, 11: 1-12.

Lourenção, A.L., V.P. Oliveira \& M.A.M. Boaventura, 1985. Danos de adultos de Epicauta atomaria (Germar, 1821) em plantas de farinha-seca. Bragantia, 44: 437-440.

Marques, A.P.C., R. Ale-Rocha \& B. Ronchi-Teles. 2004. Flutuação Populacional de Willistoniella Mik e Ropalomera Wiedemann (Diptera: Ropalomeridae) na Amazônia Central. Neotropical Entomology, 33:661-664.

Medvedev, L.N., 2004. Notes on Neotropical Alticinae (Coleoptera, Chrysomelidae). Bonner Zoologische Beitrange, 52: 121-125.

Montemayor, S.I., 2009. Description of a new Corythucha Stål from Argentina (Hemiptera: Heteroptera: Tingidae), with a description of its life cycle. Zootaxa, 2170: 61-68

Neciosup, M.A.G. \& D.P. Ojeda, 1973. Seis especies de Tingimi (Hemiptera: Tingidae) del Peru. XV Convención Nacional de Entomología, 16: 102-110.

Pahlen, A., 1977. Cubiu (Solanum topiro Humbl. \& Bonpl.), uma fruteira da Amazonia. Acta Amazonica, 7: 301-107.

Picanço, M., V.W.D. Casali, I.R. Oliveira \& G.L.D. Leite, 1997. Himenópteros associados a Solanum gilo Raddi (Solanaceae). Revista Brasileira de Zoologia, 14: 821-829.

Pires, A.M.B., P.S. Silva, P.M. Nardelli, J.C. Gomes \& A.M. Ramos, 2006. Caracterização e processamento de cubiu (Solanum sessiliflorum). Revista Ceres, 53: 309-316.

Rafael, J.A., G.A.A. Melo, C.J.B. Carvalho, S.A. Casari \& R. Constantino, 2012. Insetos do Brasil: Diversidade e Taxonomia. Ribeirão Preto, Holos, 81op.

Reis, B.L., D.C. Ferreira, R.R. Dias \& C.R. Ribas, 2001. Por que insetos podem alimentar-se de plantas tóxicas. Academia Insecta, 1: 5-7.

Rodrigues-Netto, S.M. \& D.J. Guilhem, 2000. Epicauta atomaria (Germ.) (Coleoptera, Meloidae), primeiro registro de ocorrência em pomares de maracujá (Passiflora edulis var. flavicarpa, Degener) na região Oeste do Estado de São Paulo. Arquivos do Instituto Biológico, 67: 269-270.

Rossetto, D. \& V. Nagai, 1980. Ensaio de livre escolha para o coleóptero Colaspis sp. em seis variedades de soja. Revista Cientifica do Instituto Agronômico de Campinas, 39: 195198.

Schaefer, C.W., 1998. Notes on Dysdercus from Brazil (Hemiptera: Pyrrhocoridae). Anais da Sociedade Entomológica do Brasil, 27: 485-488.

Silva-Filho, D.F., 1998. Cocona (Solanum sessiliflorum Dunal): Cultivo y utilizacion. Tratado de Cooperacion Amazonica, Secretaria Pro-Tempore, Caracas, Venezuela, 105p.

Silva-Filho, D.F., H. Noda, W.O. Paiva, K. Yuyama, C.R. Bueno \& F.M. Machado, 1997. Hortaliças não convencionais nativas e introduzidas na Amazônia, p. 19-58. In: Noda, N., L.A.G. Souza \& O.J.M. Fonseca (Eds.). Duas décadas de contribuições do INPA à pesquisa Agronômica no Trópico Úmido. Manaus, MCT-INPA, 332p.

Sosa-Gomes, D.R., B.S. Corrêa-Ferreira, C.B. Hoffmann-Campo, I.C. Corso, L.J. Oliveira, F. Moscardi, A.R. Panizzi, A.F. Bueno \& E. Hirose, 2010. Manual de Identificação de Insetos e Outros Invertebrados da Cultura da Soja. Londrina: Embrapa (Documentos, 269), 8op.

Tatu, A.I. \& I. Tausan, 2011. Corythucha ciliata (Say, 1832) (Hemiptera: Tingidae) - Second record for the lace bug fauna 
of Romania. Brukenthal. Acta Musei, 3: 453-458.

Tavares, M.T. \& B.C. Araujo, 2007. Espécies de Chalcididae (Hymenoptera, Insecta) do Estado do Espírito Santo, Brasil. Biota Neotropica, 7: 213-220.

Tisler, A.M. 1990. Feeding in the Pigweed Flea Beetle, Disonycha glabrata Fab. (Coleoptera: Chrysomelidade), on Amaranthus retroflexus. Virginia Journal of Science, 41: 243-245.

Triplehorn, C.A. \& N.F. Jonnson, 2011. Estudos dos Insetos, $7^{\text {a }}$ edição. São Paulo: Cengage Learning, 809p.

Varón, E.H., M.D. Moreira \& J.P. Corredor, 2010. Efecto de Corythucha gossypii sobre las hojas de higuerilla: criterios para su muestreo y control con insecticidas. Corpoica Ciencia Tecnologia Agropecuaria, 11: 41-47.

Yuyama, L.K.O., S.E. Barros, J.P.L. Aguiar, K. Yuyama \& D.F.
Silva-Filho, 2002. Quantificação de fibra alimentar em algumas populações de cubiu (Solanum sessiliflorum Dunal), Camu-camu (Myrciaria dúbia (HBK) Mc Vaugh) e açaí (Euterpe oleracea Mart.). Acta Amazonica, 32: 491-497.

Yuyama, L.K.O., S.H.M. Macedo, J.P.L. Aguiar, D.F. Silva-Filho, K. Yuyama, D.I.T. Fávaro \& M.B.A. Vasconcellos, 2007. Quantificação de macro e micro nutrientes em algumas etnovariedades d e cubiu (Solanum sessiliflorum Dunal). Acta Amazonica, 37: 425-30.

\section{Recebido em: 02/o2/2013}

Aceito em: 24/01/2014

\section{Como citar este artigo:}

Acioli, A.N.S., G.K.G. Costa, T.N. Moura, M.A. Guimarães, R. Almeida \& J.F. Miranda, 2014. Entomofauna Associada ao Cultivo do Cubiu (Solanum sessiliflorum Dunal) no Município de Benjamin Constant, Amazonas, Brasil. EntomoBrasilis, 7 (2): 99-105.

Acessível em: $\underline{\text { doi:10.12741/ebrasilis.v7i2.320 }}$
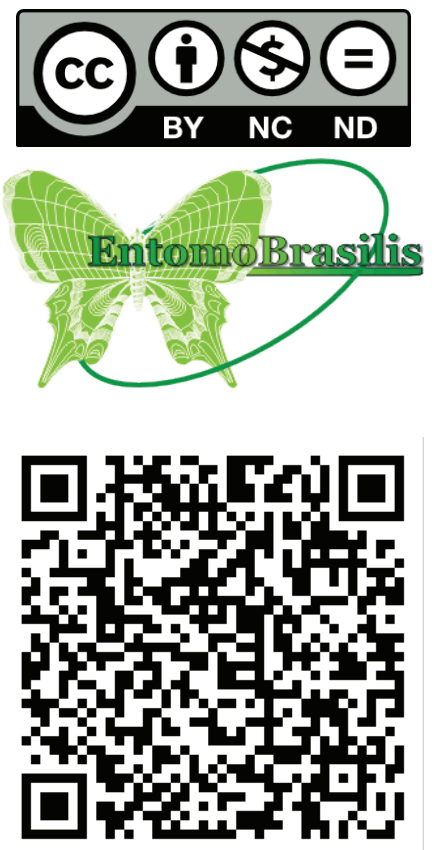